\title{
Quasi-optimal locations of piezo-elements on a rectangular plate
}

\author{
Andrzej Koszewnik ${ }^{\mathrm{a}}$ and Zdzisław Gosiewski ${ }^{\mathrm{b}}$
}

Faculty of Mechanical Engineering, Department of Automatic Control and Robotics, Bialystok University of Technology, Wiejska 45C Street, 15-351 Bialystok, Poland

Received: 25 November 2015 / Revised: 28 April 2016

Published online: 7 July 2016

(C) The Author(s) 2016. This article is published with open access at Springerlink.com

\begin{abstract}
The optimal location of piezo-actuators and piezo-sensors for the vibration control of a rectangular plate with SFSF (Simply Supported - Free - Simply Supported - Free) boundary conditions is presented in the paper. Based on bending moments, $M_{x}(x, y)$ and $M_{y}(x, y)$, the modal control forces generated by the piezo-stripes are calculated for the first five mode shapes. Calculations are carried out for different locations of two piezo-strips directed along the $X$ and $Y$ axes. The obtained results are used to define performance indexes of modal control forces for the two considered directions of vibration. In a similar way the modal unit elongations of the piezo-sensors are calculated for two different orientations of the piezos on the plate. Based on these results the objective cost functions $J_{\varepsilon \text {-odd }}$ and $J_{\varepsilon \text {-even }}$ are defined separately for odd and even modes. The quasi-optimal locations of the piezo-actuators and piezo-sensors are determined by maximizing the proposed cost functions. After analytical and numerical investigations the process of the full model identification is carried out at the laboratory stand. A chirp signal is applied in the identification process. The rectangular plate is excited with the chirp force while output signals are measured by the piezo-sensors oriented in the perpendicular directions $X$ and $Y$. In such a way two mathematical models are obtained to control the vibration of the plate separately for odd and even natural modes.
\end{abstract}

\section{Nomenclature}

$a$ - length of the plate along the $X$-direction, $\mathrm{m}$

$b$ - width of the plate along the $Y$-direction, $\mathrm{m}$

$h$ - thickness of the plate along the $Z$-direction, $\mathrm{m}$

$a_{a}$ - length of the piezo-elements (sensors and actuators) along the $X$-direction, $\mathrm{m}$

$\sigma_{f}$ - stress of the plate or piezo for bending contribution, $\mathrm{Pa}$

$\varepsilon_{f}$ - strain of the plate or piezo for bending contribution

$\sigma_{c}$ - stress of the plate or piezo for shear contribution, $\mathrm{Pa}$

$\varepsilon_{c}$ - strain of the plate or piezo for shear contribution

$\kappa$ - shear correction factor

$\xi$ - natural coordinate of the plate element along the $X$-direction

$\eta$ - natural coordinate of the plate element along the $Y$-direction

$\omega$ - natural frequency of the smart plate, $\mathrm{Hz}$

$w(x, y)$ - vertical displacement of the smart plate nodes, $\mathrm{m}$

$b_{a}$ - width of the piezo-elements along the $Y$-direction, $\mathrm{m}$

$h_{a}$ - thickness of the piezo-actuator along the $Z$-direction, $\mathrm{m}$

$h_{s}$ - thickness of the piezo-sensor along the $Z$-direction, $\mathrm{m}$

$\rho_{p}$ - plate density, $\mathrm{kg} / \mathrm{m}^{3}$

$\rho_{a}$ - piezo density, $\mathrm{kg} / \mathrm{m}^{3}$

$\varepsilon_{x}$ - total unit elongation of the piezo-sensor oriented along the $X$-direction, $\mathrm{m}$

$\varepsilon_{y}$ - total unit elongation of the piezo-sensor oriented along the $Y$-direction, $\mathrm{m}$

a e-mail: a.koszewnik@pb.edu.pl
b e-mail: z.gosiewski@pb.edu.pl 
$F_{X}$ - the modal control force generated by the piezo in the $X$-direction, N $F_{Y}$ - the modal control force generated by the piezo in the $Y$-direction, $\mathrm{N}$ $M_{x}(x, y)$ - bending moment generated by the piezo in the $X$-direction, $\mathrm{Nm}$ $M_{y}(x, y)$ - bending moment generated by the piezo in the $Y$-direction, $\mathrm{Nm}$ $U$ - voltage applied to the piezo, $\mathrm{V}$

$d_{31}, d_{32}$ - piezo-electric constants

$C_{0}$ - constant of the electro-mechanical coupling effect

$F_{a}(x, y)$ - force generated by piezo, $\mathrm{N}$

$V_{a}$ - the volume of the piezo-actuator

$V_{S}$ - the volume of the piezo-sensor

$\mathbf{D}_{f}$ - matrix of bending rigidity

$\mathbf{D}_{c}-$ matrix of shear rigidities

$\mathbf{d}_{e}-$ vector of element coordinates

$\mathbf{N}$ - matrix of Lagrange shape functions

$\mathbf{x}$ - vector of mode shapes of the smart plate

\section{Introduction}

Problems of plate vibrations are important in many structures and devices in mechanical, civil and aerospace industries. Real plates may have appreciable thickness, in which case the transverse shear and the rotary inertia are not negligible, as assumed in the classical plate theory. As a result the plate model based on the Mindlin theory has gained more popularity $[1,2]$. In recent years, the eigenvalue analyses of plates based on the Mindlin theory have been extensively investigated and new methods have been proposed.

Research on the Mindlin plate vibration can be divided into three categories. First, there exist exact solutions for a very restricted number of simple cases given by Rao [3]. Second, semi-analytical approaches are available. These include the Rayleigh-Ritz method in publications [4,5]. Finally, there are the most widely used discretization methods, such as the finite element method, the finite strip method and the difference method as can be found in the papers $[6,7]$. The development of active materials and actuators [8-10] gives new possibilities. It significantly allows us to solve the vibration damping problems of flexible structures, especially, using piezoelectric patches on their surface as actuators. The first research on application of piezoelectric materials for vibration control of structures was presented by Baily and Hubbard in 1985. They designed an active vibration damper for a vibrating beam using piezoelectric polymer actuators [11]. Next, Hagood and Crawley used piezoelectric materials and passive electrical networks to damp mechanical vibrations of space structures [12]. All the aforementioned control methods were employed for the vibration suppression of flexible structures, yet with no consideration of optimal placement of actuators as a very important problem.

In order to obtain an effective control performance, the problem of the optimal placement of actuators and sensors must be considered. Different methods and strategies are introduced in the literature to determine the optimal location of sensors and actuators. Sadri et al. suggested two criteria for the optimal placement of piezoelectric actuators using modal controllability and controllability Grammian [13]. They employed the genetic algorithm method to define the optimal location of piezoelectric actuators on the surface of a rectangular plate. Rocha et al. used the $H_{\text {inf }}$ norm to determine the optimal location of piezoelectric sensors and actuators on flexible structures [14]. Qiu et al. developed an optimal placement method of piezoelectric sensors and actuators according to the maximum observability and controllability rule by using the $\mathrm{H}_{2}$ norm [15]. On the other hand, Koszewnik and Gosiewski designed a performance index for modal control forces to determine the optimal location of piezo-strips as actuators for a beam vibration control system [16].

The calculated optimal piezo-actuator and piezo-sensor locations based on the method proposed by the authors of this paper from the control point of view allows to obtain a low-energy control system. In the case of two-dimensional structures, such as plates or shells, the control system can be described by two separate subsystems where each subsystem is related to one coordinate describing structure vibration. In most cases the location of the piezo-sensors differs from the location of the piezo-actuator. Then, such locations of the piezo-elements lead to obtaining two separate non-collocated subsystems which, next, can be translated virtually into two collocated systems. The method of the virtual collocation can be found in the papers $[17,18]$.

In this paper, the problem of the optimal location of piezo-elements for vibration control of a rectangular plate with SFSF (Simply Supported - Free - Simply Supported - Free) boundary conditions with two glued piezo-stripe actuators QP20N and two piezo-stripe sensors QP10N is investigated. The piezo-patches used in the research are elements which generate the main deformation only in one direction. So, according to the datasheet of the piezos, the voltage applied to their face can generate deformation in the longitudinal direction. Consequently, two piezo-actuators and two piezo-sensors of this type are needed to design the control vibration control system for the considered plate. 
a)

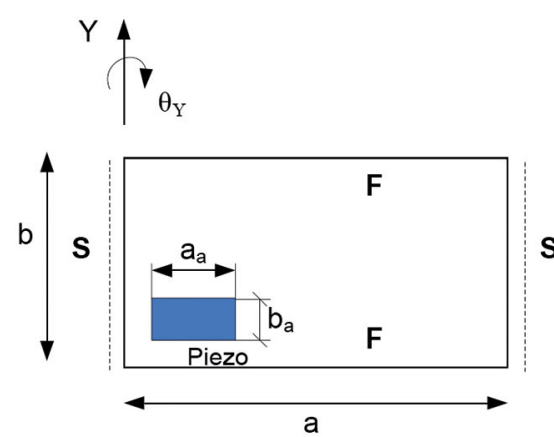

b)

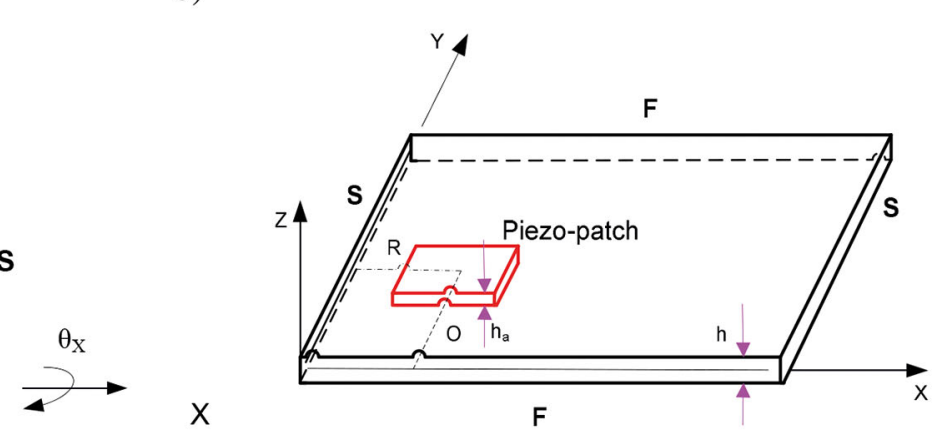

Fig. 1. Mindlin plate with single piezo-actuator: illustration of the geometry, where $\mathbf{S}$ is the simple supported edge of the plate and $\mathbf{F}$ is the free edge of the plate.

However, before the control law is designed, the model of the structure should be determined. In the considered case, the FEM model of the plate was obtained. Results of numerical simulations show that the optimal location of piezoelectric actuators and piezo-sensors depends on the sequences of the plate vibration modes. For such location of the piezo-elements on the plate the identification process is carried out at the laboratory stand. As a result of experimental investigations, two models are determined to control separately odd and even modes in the considered frequency range from 10 to $250 \mathrm{~Hz}$.

\section{Mathematical modeling}

Consider a rectangular plate of length $a$, width $b$ and height $h$ with a piezo-actuator of length $a_{a}$, width $b_{a}$ and height $h_{a}$ as shown in fig. 1. For mathematical modeling of this structure the Mindlin plate theory is used. Based on the theory, the assumed displacement field in $x, y$ and $z$ directions for the plate is defined, respectively, as [19]

$$
u=z \theta_{x} ; \quad v=z \theta_{y} ; \quad w=w_{o},
$$

where $\theta_{x}$ and $\theta_{y}$ are rotations of plate sections normal to the surface around the $y$ - and $x$-axis, respectively.

The dynamic equations of the plate with the surface bonded piezoelectric actuator are determined by using Hamilton's principle:

$$
\begin{aligned}
T-U & =W \\
T & =T_{\text {plate }}+T_{\text {piezo-act }}+T_{\text {piezo-sen }}, \\
U & =U_{\text {plate }}+U_{\text {piezo-act }}+U_{\text {piezo-sen }}
\end{aligned}
$$

where $T_{\text {plate }}, T_{\text {piezo-act }}, T_{\text {piezo-sen }}$ are the kinetic energies of the plate, the piezo-actuator and the piezo-sensor, respectively, $U_{\text {plate }}, U_{\text {piezo-act }}, U_{\text {piezo-sen }}$ are the potential energies of the plate, the piezo-actuator and the piezo-sensor, respectively, $W$ is the work done by external forces.

Taking into account eq. (2), the kinetic and potential energies for the plate and the piezo-elements are defined as

$$
\begin{aligned}
T= & \int_{V_{p}} \frac{1}{2} \rho_{p}\left(\dot{u}^{2}+\dot{v}^{2}+\dot{w}^{2}\right) \mathrm{d} V_{p}+\int_{V_{a}} \frac{1}{2} \rho_{a}\left(\dot{u}^{2}+\dot{v}^{2}+\dot{w}^{2}\right) \mathrm{d} V_{a}+\int_{V_{S}} \frac{1}{2} \rho_{S}\left(\dot{u}^{2}+\dot{v}^{2}+\dot{w}^{2}\right) \mathrm{d} V_{S} \\
U= & \int_{V_{p}} \frac{1}{2}\left\{\sigma_{f}\right\}^{T}\left\{\varepsilon_{f}\right\} \mathrm{d} V_{p}+\int_{V p} \frac{1}{2} \kappa\left\{\sigma_{c}\right\}^{T}\left\{\varepsilon_{c}\right\} \mathrm{d} V_{p}+\int_{V_{a}} \frac{1}{2}\left\{\sigma_{f}\right\}^{T}\left\{\varepsilon_{f}\right\} \mathrm{d} V_{a}+\ldots \\
& +\int_{V a} \frac{1}{2} \kappa\left\{\sigma_{c}\right\}^{T}\left\{\varepsilon_{c}\right\} \mathrm{d} V_{a}+\int_{V_{S}} \frac{1}{2}\left\{\sigma_{f}\right\}^{T}\left\{\varepsilon_{f}\right\} \mathrm{d} V_{S}+\int_{V_{S}} \frac{1}{2} \kappa\left\{\sigma_{c}\right\}^{T}\left\{\varepsilon_{c}\right\} \mathrm{d} V_{S},
\end{aligned}
$$

where $\kappa$ is the shear correction factor.

The model of the plate is discretized into several four-noded rectangular elements by using the finite element method. The length and width of the element are equal to $1 / 2$ length and $1 / 2$ width of the piezo-element. In such way, each element is defined by four nodes in natural coordinates $(\xi, \eta)$. Then, generalized displacements of the finite elements are interpolated by shape functions [19]

$$
x=\sum_{i=1}^{4} N_{i}(\xi, \eta) x_{i} \quad \text { and } \quad y=\sum_{i=1}^{4} N_{i}(\xi, \eta) y_{i},
$$


where $x_{i}, y_{i}$ are nodal displacements, for $i=1 \ldots 4, \xi$ the natural coordinate of the plate element along the $X$-direction, $\eta$ the natural coordinate of the plate element along the $Y$-direction, $N_{i}$ are the Lagrange shape functions defined as

$$
\begin{array}{ll}
N_{1}(\xi, \eta)=\frac{1}{4}(1-\xi)(1-\eta) & N_{3}(\xi, \eta)=\frac{1}{4}(1+\xi)(1+\eta) \\
N_{2}(\xi, \eta)=\frac{1}{4}(1+\xi)(1-\eta) & N_{4}(\xi, \eta)=\frac{1}{4}(1-\xi)(1+\eta) .
\end{array}
$$

The nodal displacements are used to determine strain-displacement matrices for bending and shear contribution for each element. The strain-displacement matrices are derived based on shape functions. The potential energies for the plate and for the piezos are as follows [19]:

$$
\begin{aligned}
U_{\text {plate }} & =\frac{1}{2} \mathbf{d}_{e}^{T} \int_{0}^{a_{a}} \int_{0}^{b_{a}} \int_{-\frac{h}{2}}^{\frac{h}{2}} \mathbf{B}_{f}^{T} \mathbf{D}_{f} \mathbf{B}_{f} \mathbf{d}_{e} \mathrm{~d} X \mathrm{~d} Y \mathrm{~d} Z+\frac{\kappa}{2} \mathbf{d}_{e}^{T} \int_{0}^{a_{a}} \int_{0}^{b_{a}} \int_{-\frac{h}{2}}^{\frac{h}{2}} \mathbf{B}_{c}^{T} \mathbf{D}_{c} \mathbf{B}_{c} \mathbf{d}_{e} \mathrm{~d} X \mathrm{~d} Y \mathrm{~d} Z \\
U_{\text {piezo-act }} & =\frac{1}{2} \mathbf{d}_{e}^{T} \int_{R-\frac{a_{a}}{2}}^{R+\frac{a_{a}}{2}} \int_{O-\frac{b_{a}}{2}}^{O+\frac{b_{a}}{2}} \int_{\frac{h}{2}}^{\frac{h}{2}+h_{a}} \mathbf{B}_{f}^{T} \mathbf{D}_{f}^{a} \mathbf{B}_{f} \mathbf{d}_{e} \mathrm{~d} X \mathrm{~d} Y \mathrm{~d} Z \\
U_{\text {piezo-sen }} & =\frac{1}{2} \mathbf{d}_{e}^{T} \int_{R-\frac{a_{a}}{2}}^{R+\frac{a_{a}}{2}} \int_{O-\frac{b_{a}}{2}}^{O+\frac{b_{a}}{2}} \int_{\frac{h}{2}}^{\frac{h}{2}+h_{s}} \mathbf{B}_{f}^{T} \mathbf{D}_{f}^{a} \mathbf{B}_{f} \mathbf{d}_{e} \mathrm{~d} X \mathrm{~d} Y \mathrm{~d} Z
\end{aligned}
$$

where

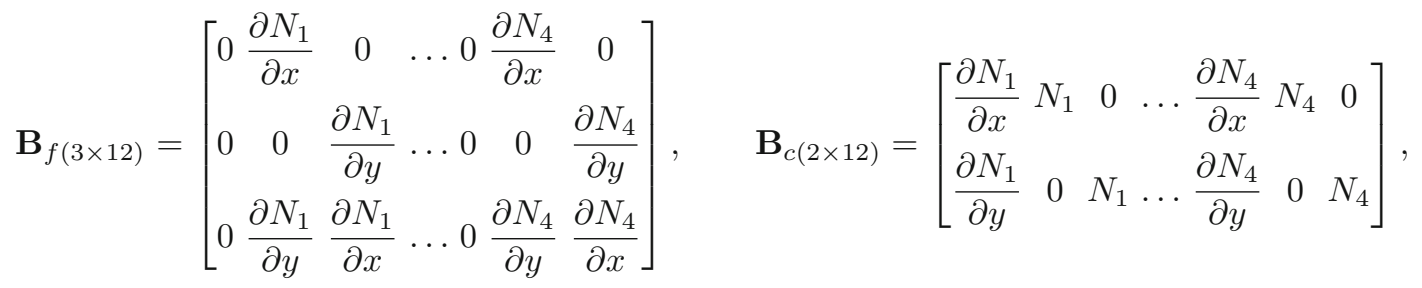

$$
\begin{aligned}
& \mathbf{D}_{f}=\frac{E}{1-v^{2}}\left[\begin{array}{ccc}
1 & v & 0 \\
v & 1 & 0 \\
0 & 0 & \frac{1-v}{2}
\end{array}\right], \quad \mathbf{D}_{f}^{a}=\frac{E}{1-v_{a}^{2}}\left[\begin{array}{ccc}
1 & v_{a} & 0 \\
v_{a} & 1 & 0 \\
0 & 0 & \frac{1-v_{a}}{2}
\end{array}\right], \\
& \mathbf{d}_{e}^{T}(1 \times 12)=\left[\begin{array}{lll}
w_{1} \theta_{x 1} & \theta_{y 1} \ldots w_{4} \theta_{x 4} \theta_{y 4}
\end{array}\right], \quad \mathbf{D}_{C}=\frac{\kappa h E}{2(1+v)}\left[\begin{array}{ll}
1 & 0 \\
0 & 1
\end{array}\right] \text {. }
\end{aligned}
$$

Finally, according to the Mindlin plate theory, the stiffness matrices of the element without and with the piezos are as follows:

$$
\mathbf{K}_{e}=\frac{h^{3}}{12} \int_{0}^{a_{a}} \int_{0}^{b_{a}} \mathbf{B}_{f}^{T} \mathbf{D}_{f} \mathbf{B}_{f} \mathrm{~d} x \mathrm{~d} y+\kappa h \int_{0}^{a_{a}} \int_{0}^{b_{a}} \mathbf{B}_{c}^{T} \mathbf{D}_{c} \mathbf{B}_{c} \mathrm{~d} x \mathrm{~d} y
$$

and

$$
\begin{aligned}
\mathbf{K}_{e}= & \frac{h^{3}}{12} \int_{0}^{a_{a}} \int_{0}^{b_{a}} \mathbf{B}_{f}^{T} \mathbf{D}_{f} \mathbf{B}_{f} \mathrm{~d} x \mathrm{~d} y+\kappa h \int_{0}^{a_{a}} \int_{0}^{b_{a}} \mathbf{B}_{c}^{T} \mathbf{D}_{c} \mathbf{B}_{c} \mathrm{~d} x \mathrm{~d} y+\frac{h_{a}^{3}}{12} \int_{R-\frac{a_{a}}{2}}^{R-\frac{a_{a}}{2}} \int_{O-\frac{b_{a}}{2}}^{O+\frac{b_{a}}{2}} \mathbf{B}_{f}^{T} \mathbf{D}_{f}^{a} \mathbf{B}_{f} \mathrm{~d} x \mathrm{~d} y \\
& +\frac{h_{s}^{3}}{12} \int_{R-\frac{a_{a}}{2}}^{R-\frac{a_{a}}{2}} \int_{O-\frac{b_{a}}{2}}^{O+\frac{b_{a}}{2}} \mathbf{B}_{f}^{T} \mathbf{D}_{f}^{a} \mathbf{B}_{f} \mathrm{~d} x \mathrm{~d} y
\end{aligned}
$$

In a similar way, by using the kinetic energy defined in eq. (3), the mass matrices of the element without and with the piezos are as follows:

$$
\mathbf{M}_{e}=\int_{0}^{a_{a}} \int_{0}^{b_{a}} \rho_{p} \mathbf{N}^{T}\left[\begin{array}{ccc}
h & 0 & 0 \\
0 & \frac{h^{3}}{12} & 0 \\
0 & 0 & \frac{h^{3}}{12}
\end{array}\right] \mathbf{N} \mathrm{d} x \mathrm{~d} y
$$


where

$$
\begin{aligned}
\mathbf{N}_{(3 \times 12)}= & {\left[\begin{array}{ccccccc}
N_{1} & 0 & 0 & \ldots & N_{4} & 0 & 0 \\
0 & N_{1} & 0 & \ldots & 0 & N_{4} & 0 \\
0 & 0 & N_{1} & \ldots & 0 & 0 & N_{4}
\end{array}\right] . } \\
\mathbf{M}_{e}= & \int_{0}^{a_{a}} \int_{0}^{b_{a}}\left(\rho_{p}\right) \mathbf{N}^{T}\left[\begin{array}{ccc}
h & 0 & 0 \\
0 & \frac{h^{3}}{12} & 0 \\
0 & 0 & \frac{h^{3}}{12}
\end{array}\right] \mathbf{N} \mathrm{d} x \mathrm{~d} y+\int_{R-\frac{a_{a}}{2}}^{R+\frac{a_{a}}{2}} \int_{O-\frac{b_{a}}{2}}^{O+\frac{b_{a}}{2}}\left(\rho_{a}\right) \mathbf{N}^{T}\left[\begin{array}{ccc}
h_{a} & 0 & 0 \\
0 & \frac{h_{a}^{3}}{12} & 0 \\
0 & 0 & \frac{h_{a}^{3}}{12}
\end{array}\right] \mathbf{N} \mathrm{d} x \mathrm{~d} y \\
& +\int_{R-\frac{a_{a}}{2}}^{R+\frac{a_{a}}{2}} \int_{O-\frac{b_{a}}{2}}^{O+\frac{b_{a}}{2}}\left(\rho_{a}\right)^{T} \mathbf{N}^{T}\left[\begin{array}{ccc}
h_{s} & 0 & 0 \\
0 & \frac{h_{s}^{3}}{12} & 0 \\
& & \\
0 & 0 & \frac{h_{s}^{3}}{12}
\end{array}\right] \mathbf{N ~ d x d y .}
\end{aligned}
$$

Taking into account boundary conditions of the smart plate ( $\mathbf{G}$, the boundary matrix) the mass and stiffness matrices of elements are transformed to the global coordinate system. The equation of motion of the plate according to the Hamilton's principle is expressed as

$$
\mathbf{M}_{G} \ddot{\mathbf{x}}+\mathbf{K}_{G} \mathbf{x}=\mathbf{0},
$$

where $\mathbf{M}_{G}=\mathbf{G}^{T} \mathbf{M}_{e} \mathbf{G}$ is the global mass matrix and $\mathbf{K}_{G}=\mathbf{G}^{T} \mathbf{K}_{e} \mathbf{G}$ is the global stiffness matrix.

Finally, assuming a harmonic motion, natural frequencies and mode shapes of the plate can be obtained by solving the eigenvalue problem,

$$
\left(\mathbf{K}_{G}-\omega^{2} \mathbf{M}_{G}\right) \mathbf{x}=\mathbf{0}
$$

where $\omega$ is a natural frequency of vibrations and $\mathbf{x}$ is a vector of the corresponding mode shape.

\section{The influence of piezo-element location on the plate natural frequencies}

A correct location of the piezo-actuator on the plate is essential for further design of low-energy control law for an active vibration damping system, especially for a few first mode shapes of the plate. The design process has many stages. In the first step, a model of the plate with size $0.4 \times 0.2 \times 0.003 \mathrm{~m}($ length $\times$ width $\times$ height $)$ is created with ANSYS. The plate is divided into 435 elements of size $25 \times 12.5 \mathrm{~mm}$ (length $\times$ width). As the result of the FEM calculations the first five natural frequencies of the plate are obtained and their modes shapes are shown in fig. 2.

The obtained results are presented in the form of the vertical deflection of the plate for the first five mode shapes. It can be seen that odd mode shapes are symmetrical along the $Y$-axis. In contrast, even modes are skew-symmetrical along the $X$-and $Y$-axis (the $X$-axis is related with the length of the plate, and the $Y$-axis is related with the width of the plate).

To find out how the location of the piezo-actuator with size $x_{a}=50 \times y_{a}=25 \times z_{a}=0.38 \mathrm{~mm}($ length $\times$ width $\times$ height) influences the values of natural frequencies and mode shapes, we consider different orientations of the piezo on the plate. At first, the actuator oriented parallel to the longitudinal edge of the plate is placed on the lower left corner of the plate (see fig. 1). Next, the piezo is moved with a step of $1 / 4$ length and $1 / 2$ width of the piezo along the whole surface of the plate. The calculations of the first five natural frequencies are repeated 90 times for different locations of the piezo on the plate. The results are shown in fig. 3.

The obtained results present a strong dependence between the location of the piezo on the plate and the mode shape, especially for even mode shapes. For even modes we can see the largest change of the even natural frequencies ( $2 \%$ in comparison with odd modes, $0.2 \%$ ). Such behavior of the plate is not unusual because the even mode shapes are simultaneously symmetrical along the $X$ - and $Y$-axes, but the odd modes are only symmetrically along the $X$-axis. Thus, in order to properly control the vibration of the plate for the even modes, there is a need to glue a second piezo to the plate oriented parallel to the $Y$-axis. In this case both piezos will generate correct bending moments in two perpendicular directions.

\section{Control forces}

In this section the rectangular plate with two piezo-actuators oriented in two perpendicular directions is investigated. At first, the aluminum plate without the piezos is considered. The motion equation of free vibrations of the plate after 


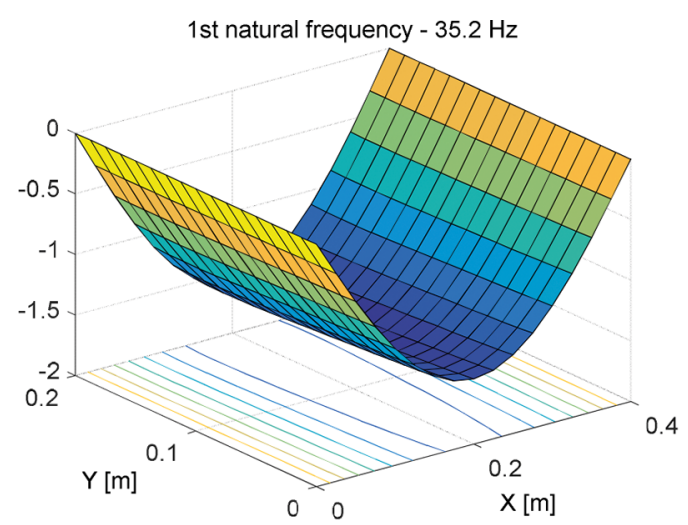

3rd natural frequency $-137.2 \mathrm{~Hz}$

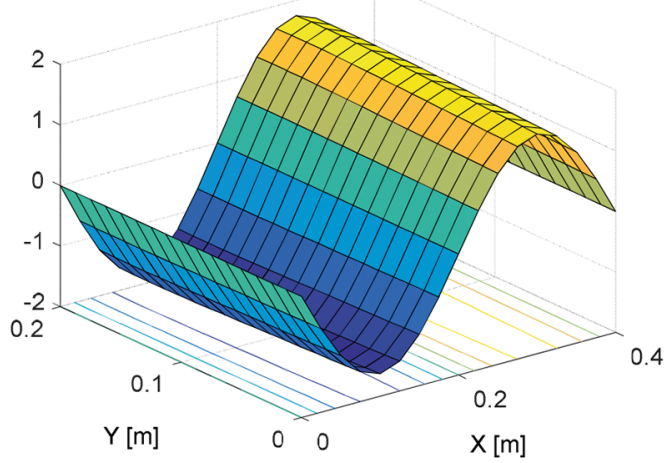

5th natural frequency $-220.6 \mathrm{~Hz}$

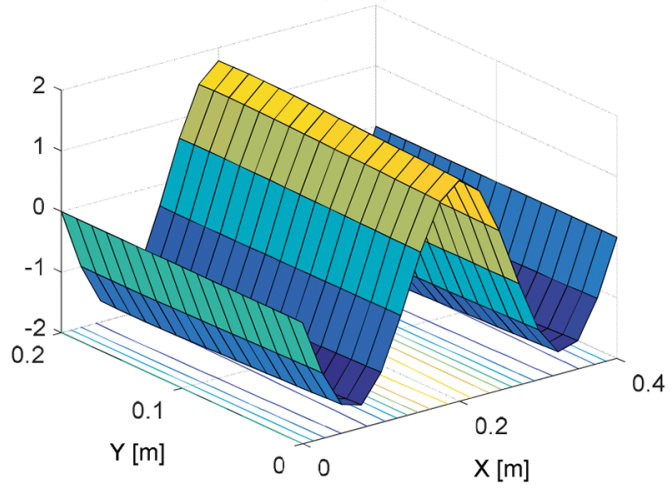

2nd natural frequency $-69.55 \mathrm{~Hz}$

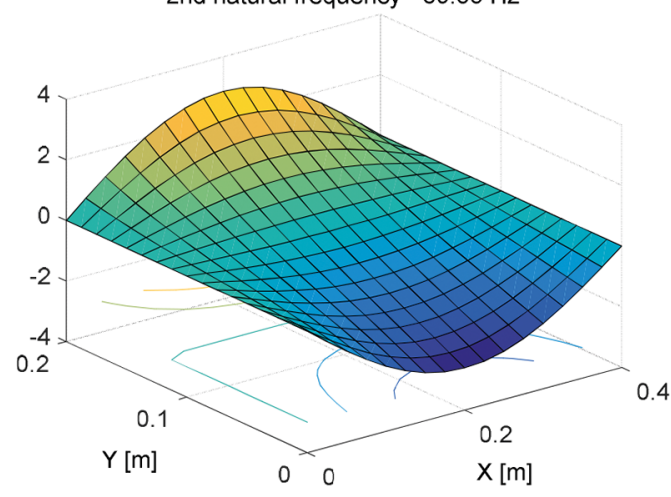

4th natural frequency $-164.5 \mathrm{~Hz}$

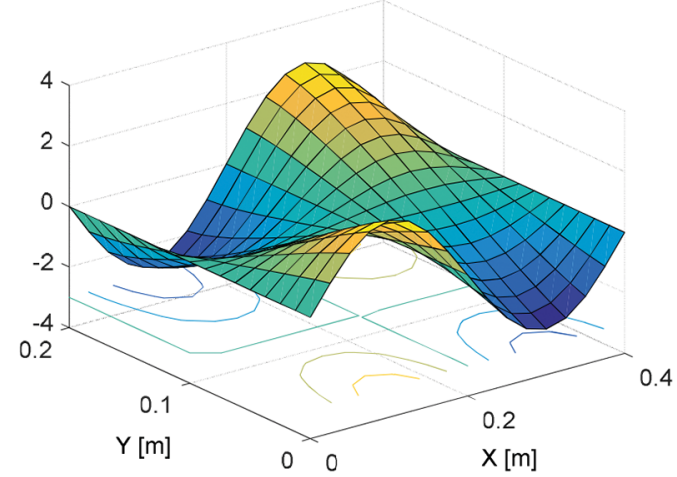

Fig. 2. The first five mode shapes of the aluminum plate.

separation of time and geometric variables can be expressed as

$$
D \Delta^{2} w(x, y)=D\left(\frac{\partial^{4} w(x, y)}{\partial x^{4}}+2 \frac{\partial^{4} w(x, y)}{\partial x^{2} \partial y^{2}}+\frac{\partial^{4} w(x, y)}{\partial y^{4}}\right)=0
$$

where $D$ is the flexural rigidity of the plate.

Taking into account the boundary conditions of the smart plate with simply supported edges (two opposite edges parallel to the $Y$-axis) vertical displacements of the plate can be presented as follows [20]:

$$
\begin{aligned}
w & =0, \quad \frac{\partial^{2} w}{\partial x^{2}}+\nu \frac{\partial^{2} w}{\partial y^{2}}=0, \quad \text { for } x=0, a \\
w(x, y) & =\sum_{n=1}^{5}\left(A_{n} \operatorname{ch} \alpha_{n} y+B_{n} \operatorname{sh} \alpha_{n} y+C_{n} \alpha_{n} \operatorname{ch} \alpha_{n} y+D_{n} \alpha_{n} \operatorname{sh} \alpha_{n} y\right) \sin \alpha_{n} x,
\end{aligned}
$$

where $\alpha_{n}=\frac{n \pi}{a}$ and $n$ is $n$-th mode shape. 

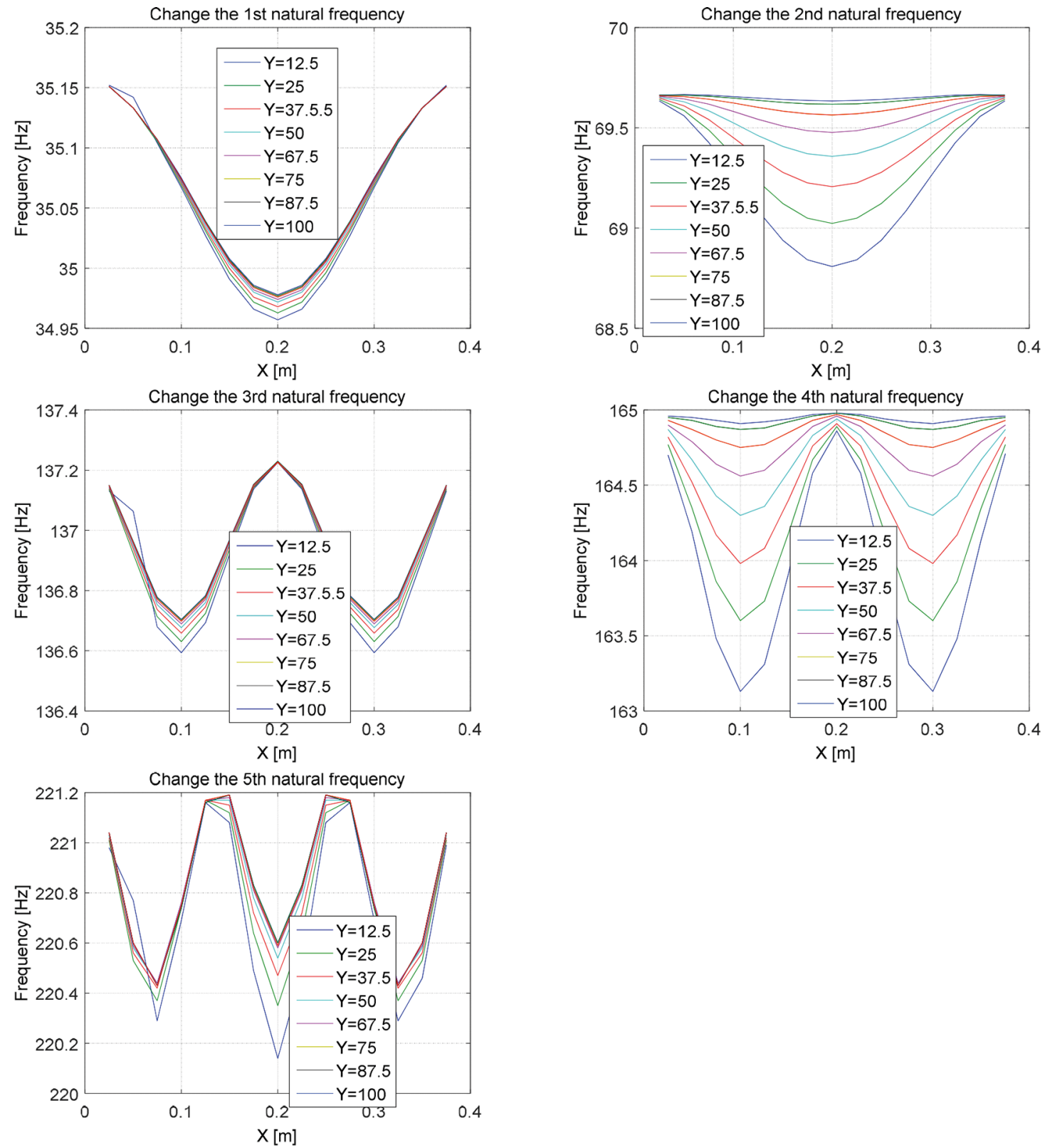

Fig. 3. The first five natural frequencies for different locations of the piezo along $X, Y$ (piezo orientated parallel to the $X$-axis as is shown in fig. 1).

Next, the plate is equipped with two piezo-actuators. The first piezo is oriented parallel to the $X$-axis while the second is parallel to the $Y$-axis. Both piezos generate bending moments, $M_{x}(x, y)$ and $M_{y}(x, y)$, in two perpendicular directions, so they influence the vibrations of the whole plate. The equation of motion of the smart plate (with piezos) is developed in the following form:

$$
\frac{\partial^{4} w(x, y)}{\partial x^{4}}+2 \frac{\partial^{4} w(x, y)}{\partial x^{2} \partial y^{2}}+\frac{\partial^{4} w(x, y)}{\partial y^{4}}=\frac{\partial^{2} M_{x}(x, y)}{\partial x^{2}}+\frac{\partial^{2} M_{y}(x, y)}{\partial y^{2}}
$$

Bending moments $M_{x}(x, y)$ and $M_{y}(x, y)$ in eq. (18) represent force excitation. Taking into account the orientation of the piezos on the plate, each moment can be defined with control forces generated by individual piezo (see fig. 4) and with the electro-mechanical coupling effect. In our case, the distributed control forces are expressed with the point 
a)

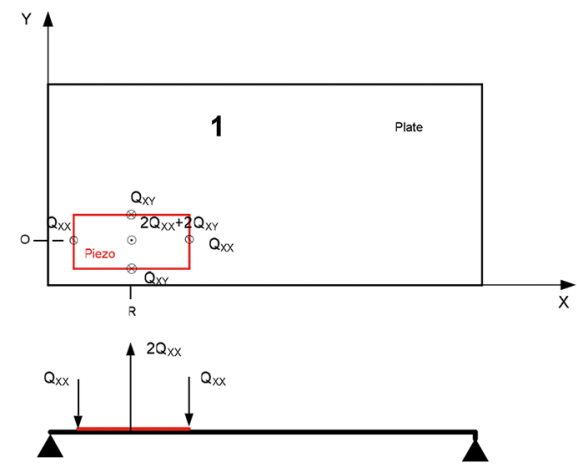

b)

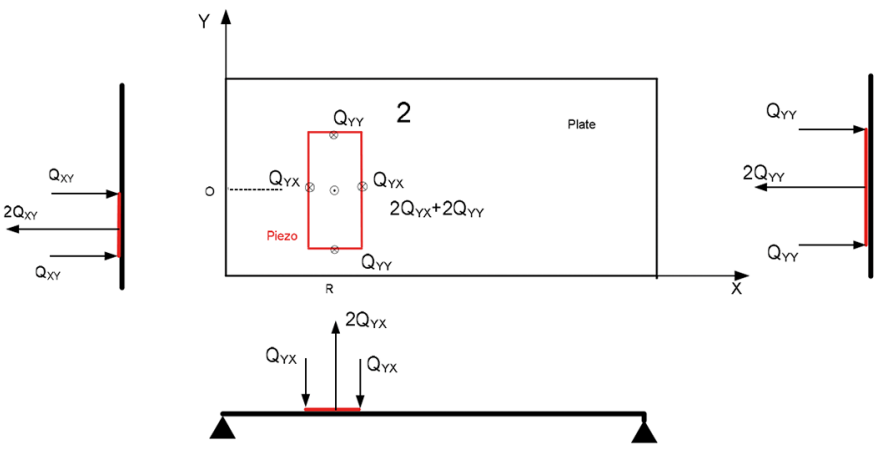

Fig. 4. Distribution of control forces on the plate piezo (a) parallel to the $X$-axis and (b) parallel to the $Y$-axis.

forces as is shown in fig. 4 . Thus, the bending moments $M_{x}(x, y)$ and $M_{y}(x, y)$ take the form

$$
\begin{aligned}
& \left\{\begin{array}{l}
M_{x}(x, y) \\
M_{y}(x, y)
\end{array}\right\}=C_{0} \cdot \frac{U}{h_{a}} \cdot\left[\begin{array}{ll}
Q_{X X} & Q_{Y Y} \\
Q_{Y X} & Q_{X Y}
\end{array}\right] \cdot\left\{\begin{array}{l}
d_{31} \\
d_{32}
\end{array}\right\} \\
& =C_{0} \cdot \frac{U}{h_{a}} \cdot\left[\begin{array}{l}
\left.Q_{X X}\left(-\delta\left(x-R-\frac{a_{a}}{2}\right)-\delta\left(x-R+\frac{a_{a}}{2}\right)+2 \delta(x-R)\right) \quad Q_{Y Y}\left(-\delta\left(y-O-\frac{b_{a}}{2}\right)-\delta\left(y-O+\frac{b_{a}}{2}\right)+2 \delta(y-O)\right)\right] \\
Q_{Y X}\left(-\delta\left(y-R-\frac{a_{a}}{2}\right)-\delta\left(y-R+\frac{a_{a}}{2}\right)+2 \delta(y-R)\right)
\end{array} Q_{X Y}\left(-\delta\left(y-O-\frac{b_{a}}{2}\right)-\delta\left(y-O+\frac{b_{a}}{2}\right)+2 \delta(y-O)\right)\right] \\
& \cdot\left\{\begin{array}{l}
d_{31} \\
d_{32}
\end{array}\right\},
\end{aligned}
$$

where $\delta$ is the Dirac's function, $U$ is the voltage applied to the piezo, $h_{a}$ is the thickness of the piezo-actuator, $d_{31}$, $d_{32}$ the piezo-electric constants [21] and $C_{0}$ is the constant of the electro-mechanical coupling effect [21].

The constant of the electromechanical coupling effect is calculated as follows [22]:

$$
C_{0}=-\frac{E_{a}}{6} \cdot \frac{\left(1+\nu_{a}\right)}{(1-\nu)} \cdot \frac{P h^{2}}{1+\nu_{a}-\left(1+\nu_{a}\right) P}
$$

where $\nu$ is the Poisson coefficient of the plate, $\nu_{a}$ is the Poisson coefficient of the piezo-actuator, $E$ is the Young modulus of the plate, $E_{a}$ is the Young modulus of the piezo-actuator, $h$ is the thickness of the plate, $P$ is the constant defined as $P=\frac{E_{a}}{E} \cdot \frac{1-\nu^{2}}{1-\nu_{a}^{2}}$.

\section{Numerical calculations of control forces}

Numerical simulations are carried out to evaluate the ability of piezoelectric patches to generate proper forces for the active vibration reduction of the rectangular plate. For this purpose, the smart plate with SFSF boundary conditions formulated in sect. 4 was used for investigations. Since the simultaneous vibration reduction in two perpendicular directions of the plate for the first five lowest modes is considered, the numerical research is carried out in two stages. First, the piezo is oriented parallel to the longitudinal edge of the plate and next it is moved in two directions with a step of $1 / 4$ length of the piezo in the $X$-direction and $1 / 2$ width in the $Y$-direction. As a result of this procedure, 435 different locations of the piezo on the plate are obtained. For each location of the piezo, the control forces are calculated and the results are shown in fig. 5 .

Next, the procedure is repeated for the piezo-actuator oriented parallel to the $Y$-axis. Thus, the control forces in two perpendicular directions at 403 different locations of the piezo are obtained. The results for this step are presented in fig. 6.

The calculated control forces generated by the piezo in the two perpendicular directions $X$ (upper plot) and $Y$ (lower plot) for the consider mode shapes are shown in fig. 5. The values of the modal control forces for the first mode are shown in the upper left corner while the control forces for the fifth mode shape are given at the lower left corner. 

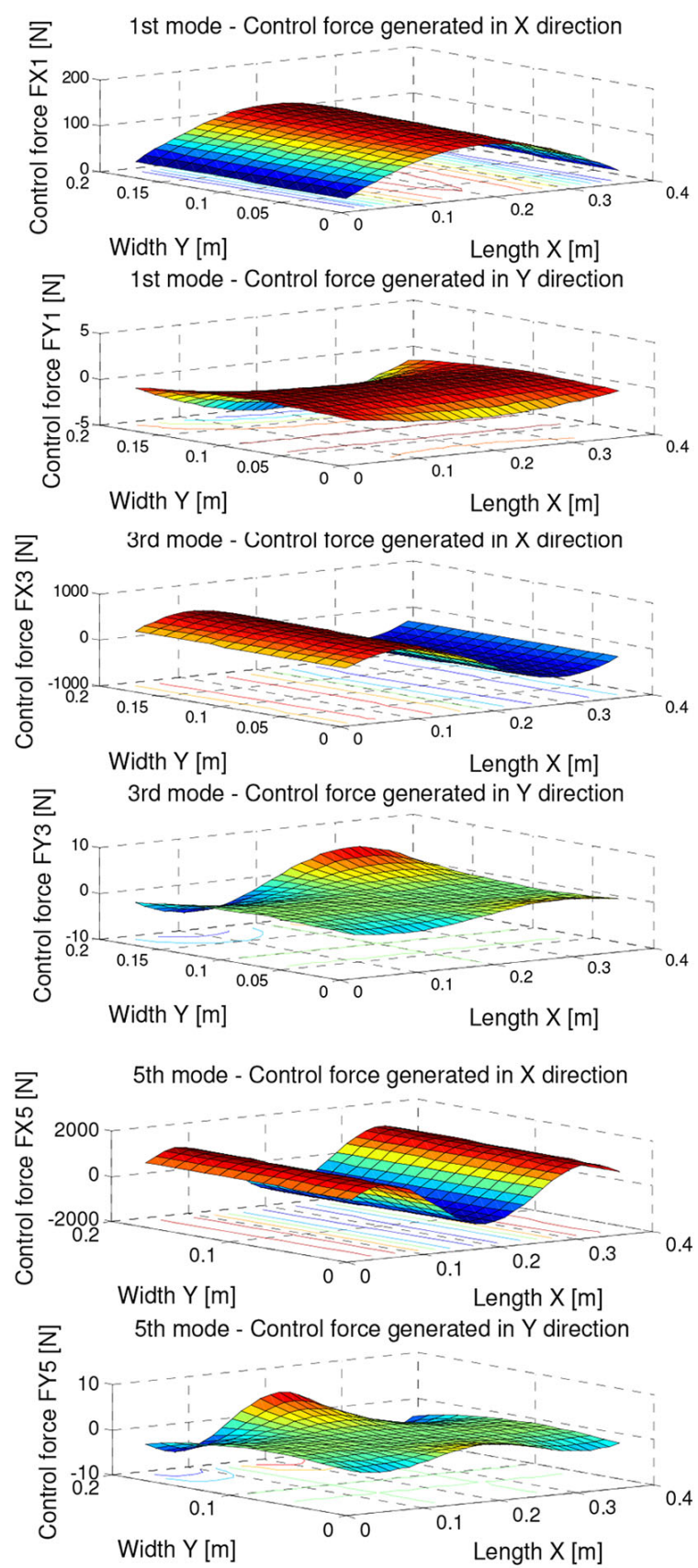
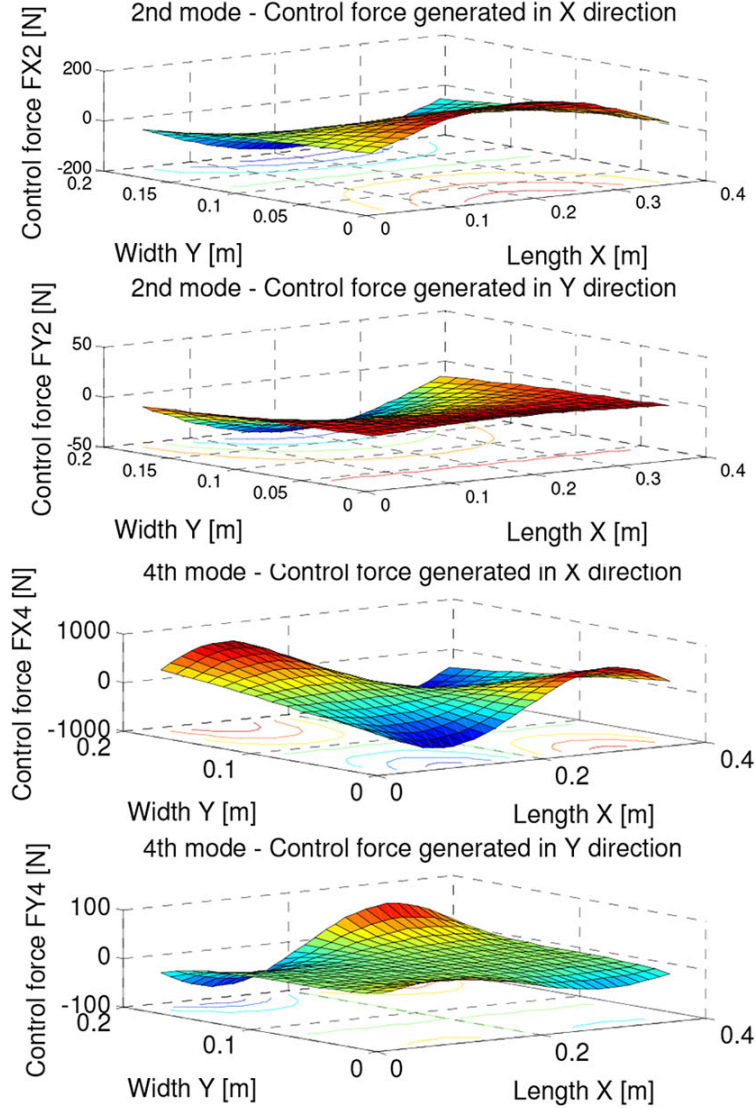

Fig. 5. Control forces generated by the piezo in the two perpendicular directions $X$ and $Y$ versus its location (piezo orientated parallel to the $X$-axis).

Comparing fig. 5 with fig. 2 one can suppose that the control forces generated by the piezo in the $X$-direction depend on the mode shapes of the plate. Thus, the modal control forces $F_{X}$ (see the upper plot) are significantly higher than the control forces $F_{Y}$ generated in the perpendicular direction. Moreover, the calculated control forces achieve their extreme (minimum and maximum) values in the vicinity of the corresponding extreme of the vertical deflection of the plate. However, in this case, the sign of the control forces is opposite to the sign of the vertical deflection of the plate. Such behavior is not unusual, because the considered plate has been simply supported at two opposite edges parallel to the $Y$-axis.

For the piezo-actuator oriented parallel to the $Y$-axis (see fig. 6) similar results are obtained. In this case the control forces $F_{X}$ are still higher than the control forces $F_{Y}$, but values of the control force $F_{Y}$ are at least twice bigger than the values of the control force $F_{Y}$ for the piezo oriented parallel to the $Y$ edge of the plate (see table 1). 

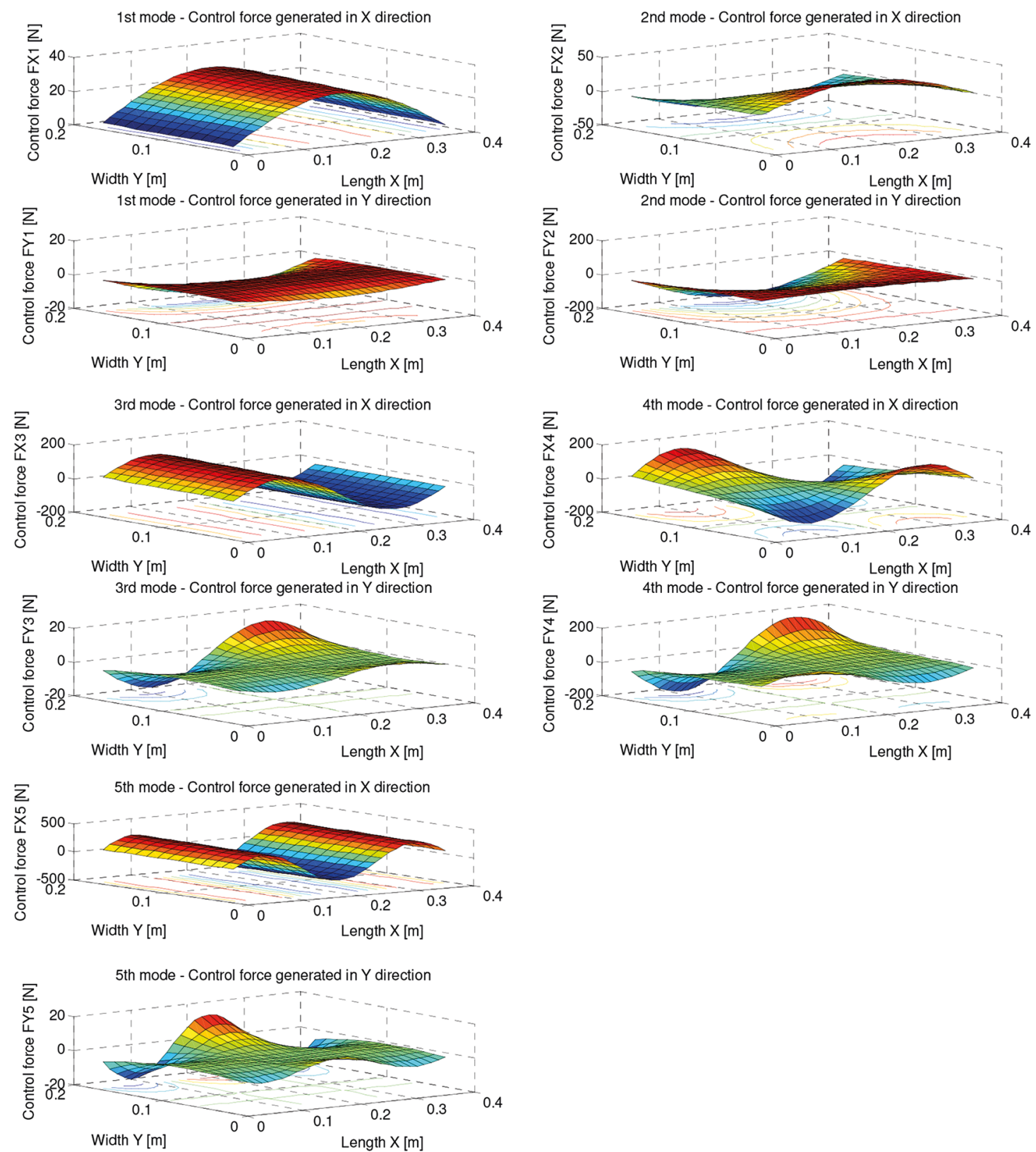

Fig. 6. Control forces generated by the piezo in the two perpendicular directions $X$ and $Y$ versus its location (piezo orientated parallel to the $Y$-axis).

Table 1. The ratio of the control force $F_{Y}$ versus orientation of the piezo-actuator on the plate. $F_{Y(X)}$ is the calculated control force $F_{Y}$ for the piezo-actuator oriented parallel to the $X$ edge of the plate and $F_{Y(Y)}$ is the calculated control force $F_{Y}$ for the piezo-actuator oriented parallel to the $Y$ edge of the plate.

\begin{tabular}{|c|c|}
\hline Mode of the smart plate & Ratio $F_{Y}=F_{Y(Y)} / F_{Y(X)}$ \\
\hline 1st mode & 4 \\
\hline 2nd mode & 4 \\
\hline 3th mode & 2 \\
\hline 4th mode & 2 \\
\hline 5th mode & 2 \\
\hline
\end{tabular}


a)

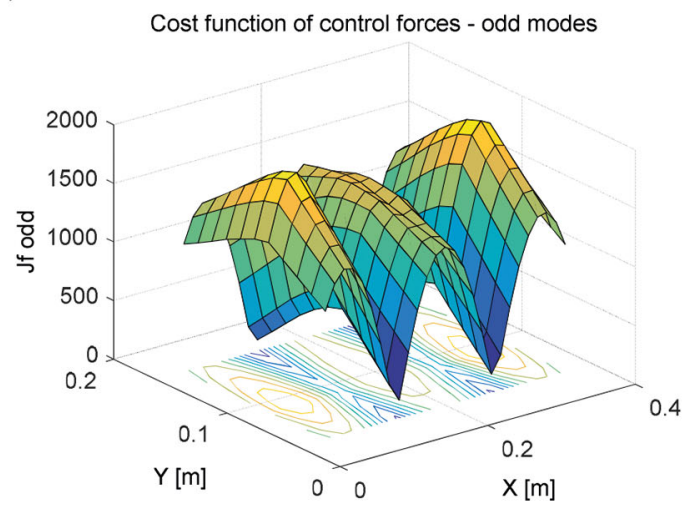

b)

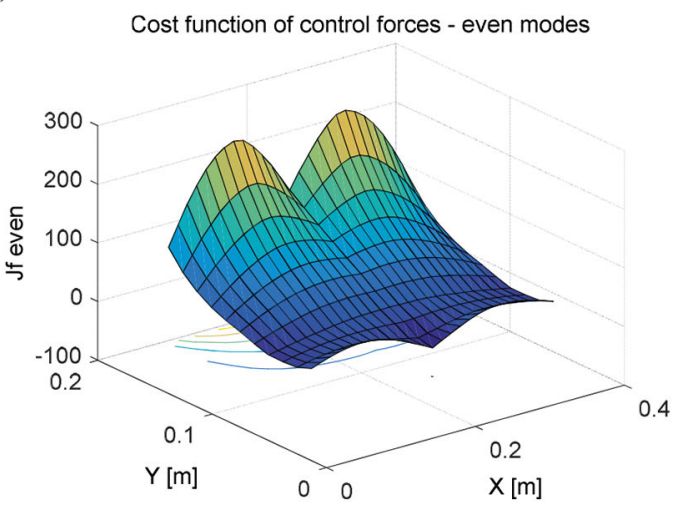

Fig. 7. Cost functions derived for modal forces versus piezo-patch locations.

\section{Optimal location of the piezo-actuators}

At this step of the present study the following question arises: how can one determine the quasi-optimal locations of the piezo-actuators on the plate? To answer this question the following two cost functions are introduced for two different orientations of the piezo-actuators on the plate, separately for odd and even modes:

$$
\begin{aligned}
J_{F-\text { odd }} & =\sum_{n=1}^{n=c m \text {-odd }}\left|F_{X}\right|-\sum_{m=1}^{m=u c m \text {-even }}\left|F_{X}\right| \\
J_{F-\text { even }} & =\sum_{n=1}^{n=c m \text {-even }}\left|F_{Y}\right|-\sum_{m=1}^{m=u c m \text {-odd }}\left|F_{Y}\right|
\end{aligned}
$$

where $n$ is the controlled modes, $m$ is the uncontrolled modes, $\mathrm{cm}$-odd is the number of considered controlled odd modes, $u \mathrm{~cm}$-even is the number of considered uncontrolled even modes, $\mathrm{cm}$-even is the number of considered controlled even modes, $u c m$-odd is the number of considered uncontrolled odd modes, $F_{X}$ is the modal control force generated by the piezo in the $X$-direction $(\mathrm{N})$ and $F_{Y}$ is the modal control force generated by the piezo in the $Y$-direction $(\mathrm{N})$.

The cost functions depend on the orientations of the actuators on the plate. If the piezo-actuator is utilized parallel to the $X$-axis then the first three odd modes are controlled while the second and fourth modes are not controlled. Then eq. (21) is used. On the other hand, if the piezo-actuator parallel to the $Y$-axis is utilized, then the first two odd modes are controlled while the first three odd modes are not controlled. In this case eq. (22) is used. The calculations of the cost functions are carried out for different locations of the piezo-actuators on the plate. During calculations boundary locations of the piezos on the plate are not included. Moreover, in order to eliminate the influence of the bending moment, $M_{x}(x, y)$, generated by the piezo oriented parallel to the $X$-axis on the bending moment, $M_{y}(x, y)$, generated by the actuator utilized parallel to the $Y$-axis, the condition of the minimum displacement between both piezos is introduced (see eq. (23)). As a result of the calculations, the values of cost functions $J_{f \text {-odd }}$ and $J_{f \text {-even }}$ are presented in fig. 7:

$$
d>\frac{a_{a}}{2}+\frac{b_{a}}{2}
$$

where $a_{a}$ is the length of the piezo-actuator, $b_{a}$ is the width of the piezo-actuator.

The shapes of the obtained modal control forces are similar to the shapes of the vertical deflections of the plate. Thus, the values of the cost functions have their extreme values at the vicinities of the minimum and maximum

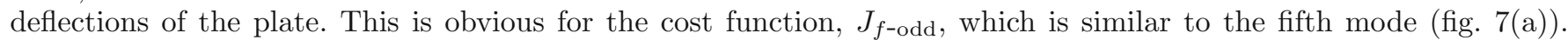

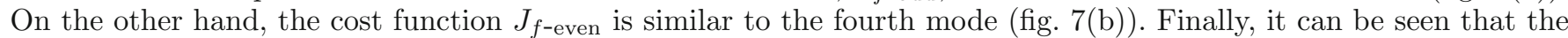
quasi-optimal locations of the actuators depend on number of the considered odd and even natural frequencies and the locations of the piezo-element on the plate.

It can be seen in fig. 7 (a) that the cost function $J_{f \text {-odd }}$ achieves its maximum values at the points $A_{x}=(0.125,0.1)$,

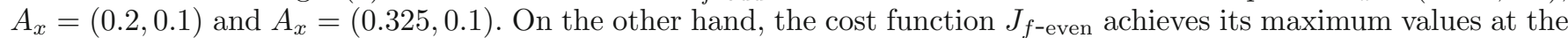
points $A_{y}=(0.15,0.1625)$ and $A_{y}=(0.325,0.1625)$. Thus, the indicated locations or their vicinity can be quasi-optimal locations of both piezo-actuators. 
a)

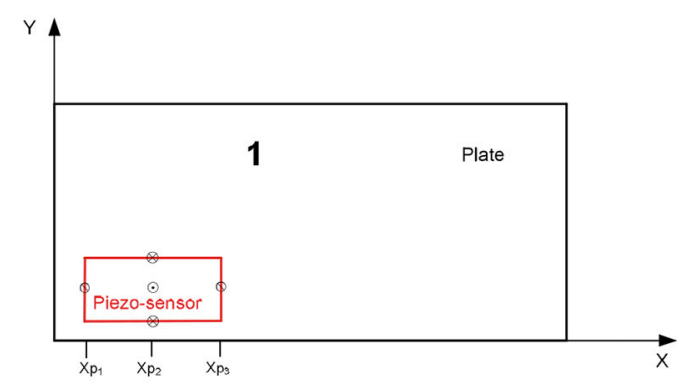

b)

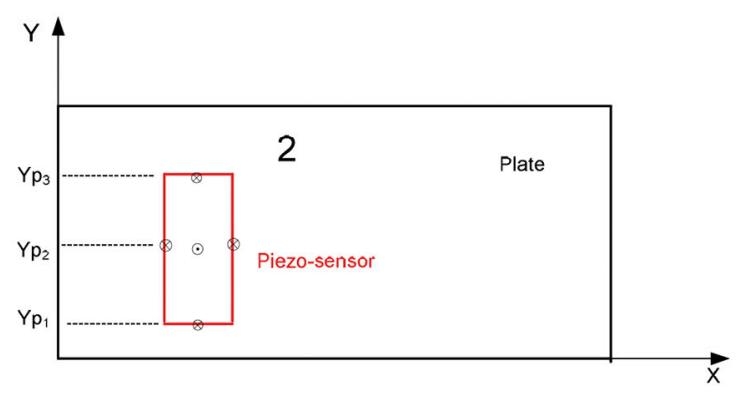

Fig. 8. The piezo-sensor divided into two parts in different orientations on the plate.

\section{Numerical calculations of the measurement signals and the optimal locations of the piezo-sensors}

It is well known that the locations of sensors have a big influence on the vibration control of such systems as plates or membranes with many node lines. To find the proper location, the elongations of the piezo-sensors have been calculated. These elongations depend on voltage signals generated on the walls of the piezos. At first we assume that the piezo-sensor is oriented parallel to the $X$-axis (fig. 8(a)). The elongation results from the angular deformation of the piezo. The angular deformation of the piezo is considered separately for its two parts indicated in fig. 8. Thus, the unit elongation of each part of the piezo is expressed by these angular deformations in the following form:

$$
\begin{aligned}
& \varepsilon_{X 1}=-\frac{\Delta r}{a_{a}}\left(\left.\frac{\partial^{2} w(x, y)}{\partial x^{2}}\right|_{x p 2}-\left.\frac{\partial^{2} w(x, y)}{\partial x^{2}}\right|_{x p 1}\right) \\
& \varepsilon_{X 2}=-\frac{\Delta r}{a_{a}}\left(\left.\frac{\partial^{2} w(x, y)}{\partial x^{2}}\right|_{x p 3}-\left.\frac{\partial^{2} w(x, y)}{\partial x^{2}}\right|_{x p 2}\right) .
\end{aligned}
$$

The total unit elongation is a sum of the unit elongations of both parts of the piezo-sensor,

$$
\varepsilon_{X}=\varepsilon_{X 1}+\varepsilon_{X 2}
$$

where $w(x, y)$ is the vertical displacement of nodes of the smart plate, $a_{a}$ is the length of the piezo-sensor along the $X$-direction, $\Delta r$ is the radius of the plate curvature in the $X$-direction, where $\Delta r=\frac{h}{2}+\frac{h_{s}}{2}$.

In the next step, we assume that the piezo-sensor is oriented parallel to the $Y$-direction. Then, the total unit elongation is expressed in the following form:

$$
\varepsilon_{Y}=\varepsilon_{Y 1}+\varepsilon_{Y 2}
$$

where

$$
\varepsilon_{Y 1}=-\frac{\Delta r}{a_{a}}\left(\left.\frac{\partial^{2} w(x, y)}{\partial y^{2}}\right|_{y p 2}-\left.\frac{\partial^{2} w(x, y)}{\partial y^{2}}\right|_{y p 1}\right) \quad \text { and } \quad \varepsilon_{Y 2}=-\frac{\Delta r}{a_{a}}\left(\left.\frac{\partial^{2} w(x, y)}{\partial y^{2}}\right|_{y p 3}-\left.\frac{\partial^{2} w(x, y)}{\partial y^{2}}\right|_{y p 2}\right)
$$

The numerical simulations are repeated to evaluate the ability of the piezoelectric patch to generate the elongations separately for two different orientations of the piezo-sensor on the plate. For this purpose the plate with the quick-pack piezo-sensor (Mide QP10N) is analyzed in two steps. At first, the piezo is oriented parallel to the longitudinal edge of the plate and, secondly, the piezo is oriented parallel to the crosswise edge. In both cases the piezo is moved in two directions with a step of $1 / 2$ length of the piezo in the $X$-direction and $1 / 2$ width in the $Y$-direction. As a result, the unit elongations $\varepsilon_{x}$ for odd modes and $\varepsilon_{y}$ for even modes are obtained, respectively. The results of these calculations are shown in fig. 9 .

As we can see in fig. 9, the modal unit elongation of the piezo-sensor strongly depends on the deflection of the plate. It is evident especially for odd modes when the piezo is oriented parallel to the $X$-axis. Then, the minimum unit elongations $\varepsilon_{X}$ are obtained exactly at the same locations of minimum deflections of the plate. Additionally, the unit elongations $\varepsilon_{X}$ are obtained also at locations of nodal lines of vertical deflections of the plate. 

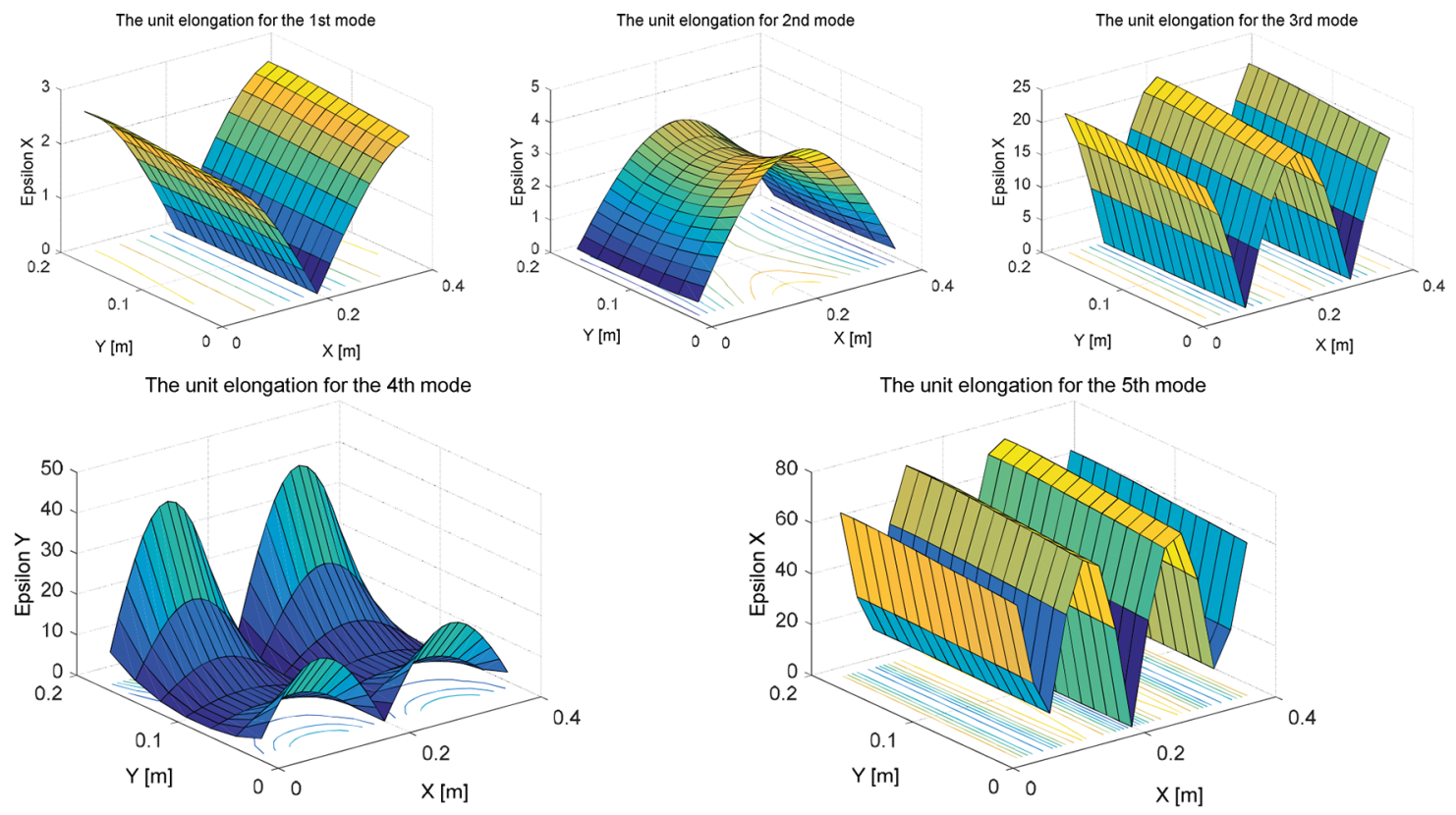

Fig. 9. Absolute values of modal unit elongation versus piezo-sensor location and orientation for the five lowest modes.

a)

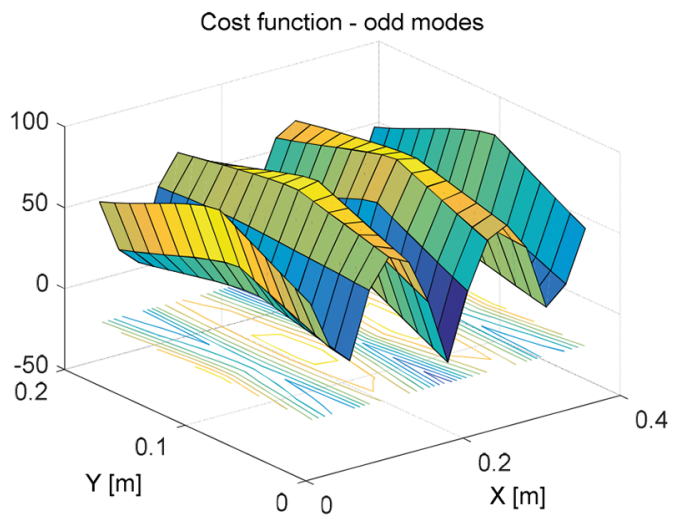

b)

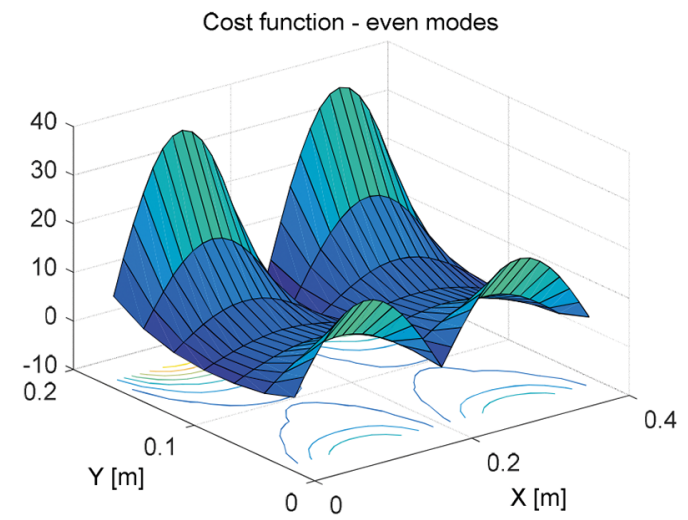

Fig. 10. Cost functions obtained for different piezo-sensors locations on the plate (a) parallel to the $X$-axis, (b) parallel to the $Y$-axis.

The obtained results can be used to find the best location of the piezo-sensors on the plate. For this purpose the following cost functions are defined separately for odd and even modes,

$$
\begin{aligned}
J_{\varepsilon \text {-odd }} & =\sum_{n=1}^{n=c m \text {-odd }}\left|\varepsilon_{X}\right|-\sum_{m=1}^{m=u c m \text {-even }}\left|\varepsilon_{X}\right| \\
J_{\varepsilon \text {-even }} & =\sum_{n=1}^{n=c m \text {-even }}\left|\varepsilon_{Y}\right|-\sum_{m=1}^{m=u c m \text {-odd }}\left|\varepsilon_{Y}\right|,
\end{aligned}
$$

where $n$ is the controlled modes, $m$ is the uncontrolled modes, $\mathrm{cm}$-odd is the number of considered controlled odd modes, $u c m$-even is the number of considered uncontrolled even modes, $\mathrm{cm}$-even is the number of considered controlled even modes, $u \mathrm{~cm}$-odd is the number of considered uncontrolled odd modes, $\varepsilon_{X}$ is the total unit elongation of the piezosensor in the $X$-direction, $\varepsilon_{Y}$ is the total unit elongation of the piezo-sensor in the $Y$-direction.

If the piezo-sensor parallel to the $X$-axis is utilized, then the first, third and fifth modes are controlled while the second and fourth modes are not controlled. In this case eq. (28) is used. On the other hand, if the piezo-sensor parallel to the $Y$-axis is utilized, then the second and fourth modes are controlled while the first, third and fifth modes are not controlled. Then eq. $(29)$ is used. The obtained values of cost functions $J_{\varepsilon \text {-odd }}$ and $J_{\varepsilon \text {-even }}$ are presented in fig. 10. 
a)

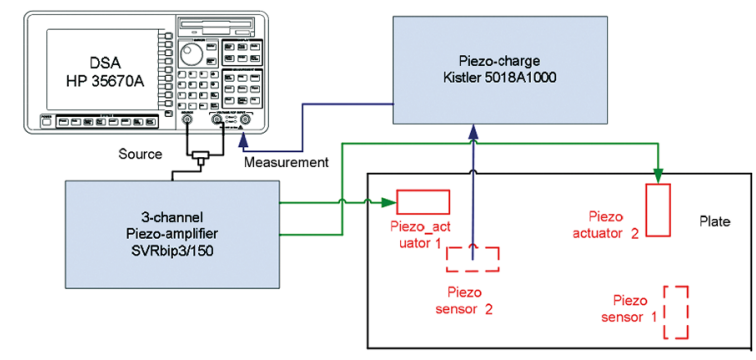

b)

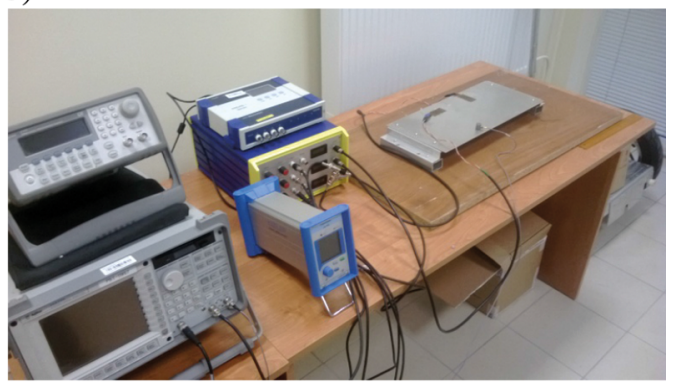

Fig. 11. Test rig (a) block diagram, (b) photo.

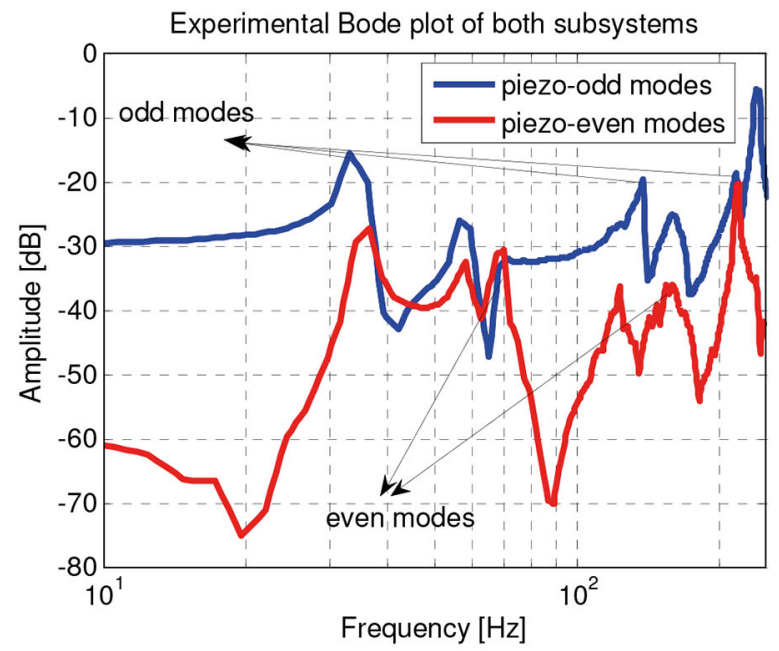

\begin{tabular}{|c|c|c|}
\hline \multicolumn{3}{|c|}{ Results of natural frequency with the } \\
\hline & $\begin{array}{c}\text { FEM method } \\
{[\mathrm{Hz}]}\end{array}$ & $\begin{array}{c}\text { Exp. } \\
\text { Identification } \\
{[\mathrm{Hz}]}\end{array}$ \\
\hline $1^{\text {st }}$ mode & 35.2 & 34.8 \\
\hline $2^{\text {nd }}$ mode & 69.55 & 69.2 \\
\hline $3^{\text {rd }}$ mode & 137.2 & 137.6 \\
\hline $4^{\text {th }}$ mode & 164.5 & 163.1 \\
\hline $5^{\text {th }}$ mode & 220.6 & 218.9 \\
\hline
\end{tabular}

Fig. 12. Experimental Bode plots of both subsystems: odd modes and even modes.

Based on the above calculations the best piezo-sensors locations can be determined to properly control the odd and even modes. In both cases a maximum of the cost functions $J_{\varepsilon \text {-odd }}$ and $J_{\varepsilon \text {-even }}$ should be defined. As a result, for the piezo-patch orientated parallel to the $X$-axis the best piezo-sensor locations on the plate are obtained at the points $S_{x}=(0.150,0.1)$ and $S_{x}=(0.275,0.1)$ (fig. 10(a)). On the other hand, for the piezo-sensor location parallel to the $Y$-axis the best piezo-sensor locations on the plate are obtained at the points $S_{y}=(0.0875,0.1625)$ and $S_{y}=(0.2875,0.1625)$ (fig. 10(b)). Similarly, the indicated locations are quasi-optimal locations of both piezo-sensors. Thus, the piezos located in these points are used in experimental tests.

\section{Identification of dynamic parameters of the plate}

This section of the paper is mainly related to the experimental identification of the plate which is an important step for the proper design of the control system. In order to determine mathematical models used to separately control the odd and even mode shapes the plate is equipped with two piezo-patch actuators and two piezo-patch sensors oriented in the perpendicular directions $X$ and $Y$. Moreover, the aforementioned components of the control system are located at opposite planes of the plate at their quasi-optimal locations. Their locations and orientations lead to consider two separate non-collocated subsystems, of which each subsystem can next be translated virtually into collocated systems. The obtained models in the mentioned way can be used to design low-energy control systems.

The full identification process was carried out at the laboratory stand (fig. 11) for both orientations of the piezosensors using the Dynamic Signal Analyzer (DSA), the 3-channel piezo-amplifier Piezomechanik SVRbip3/150 and the piezo-charge amplifier Kistler 5018A1000. The DSA generates the chirp signal $u(t)=5 \sin (w t)$ in the frequency range from $10 \mathrm{~Hz}$ to $250 \mathrm{~Hz}$. The selected frequency range from $10 \mathrm{~Hz}$ to $250 \mathrm{~Hz}$ contains the first five natural frequencies of the plate. The amplified periodic signal $u(t)$ is applied simultaneously to both piezo-actuators. The vibration of the plate is measured by two piezo-sensors. For the piezo-sensor oriented parallel to the $X$-axis, the amplitude plot of the odd modes is obtained. On the other hand, for the piezo-sensor oriented parallel to the $Y$-axis the amplitude plot of the even modes is obtained. The results of the experiment are shown in fig. 12. 

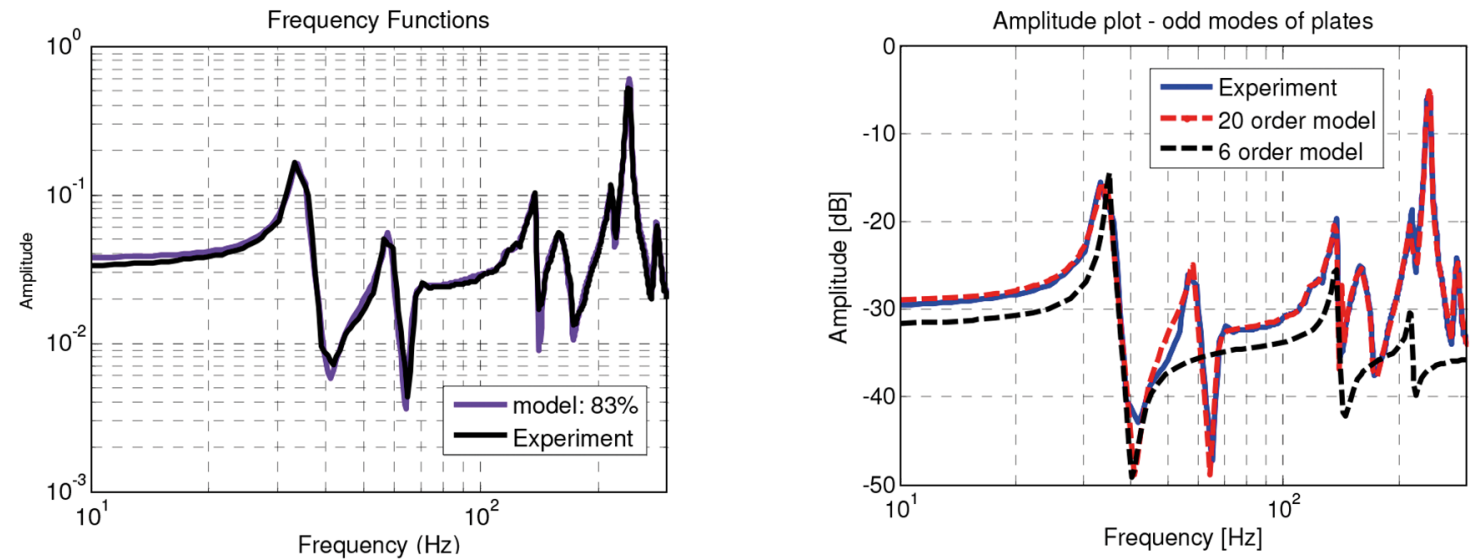

Fig. 13. Comparison of the model and the experimental data (a) 55th order model, (b) 20th order model and 6th order model.
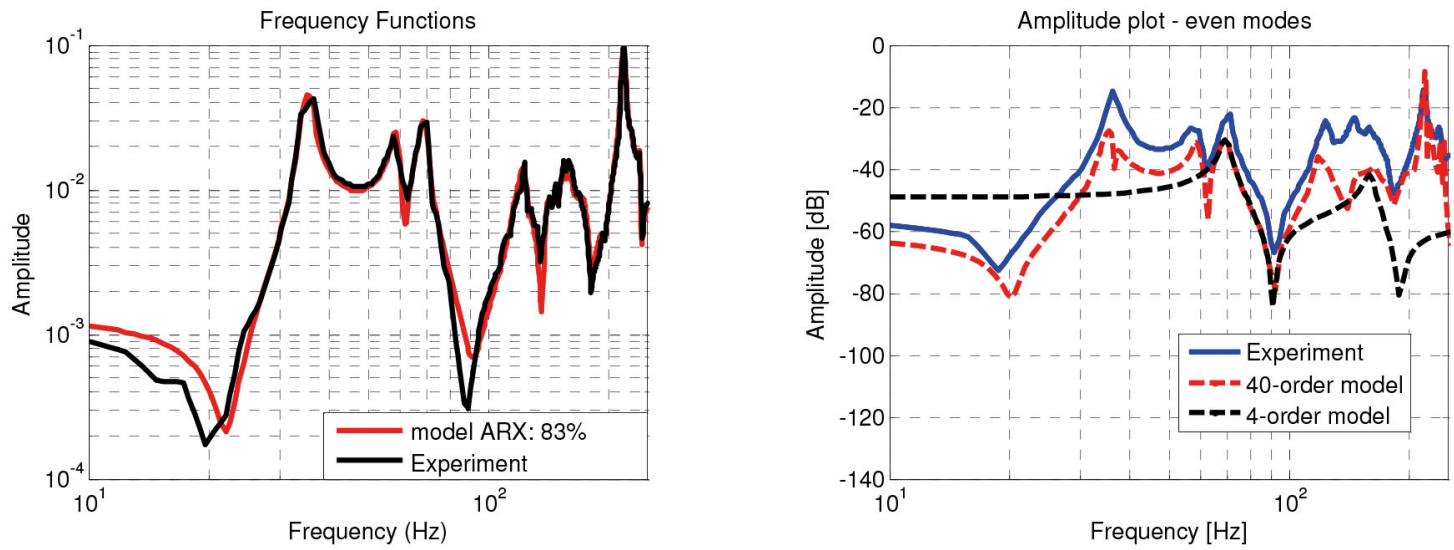

Fig. 14. Comparison of the model and the experimental data (a) 65th order model, (b) 40th order model and 4th order model.

As we can see in fig. 12 experimental values of natural frequencies of the plate are almost the same as the natural frequencies obtained with FEM calculations. Additionally, it is shown that the second resonance frequency $\left(f_{2}=69 \mathrm{~Hz}\right)$ is measured only by the piezo-sensor oriented parallel to the $Y$-axis. Thus, the proper measurement of vibrations of the plate depends on the locations of the piezo-sensor. Further analysis of both amplitude plots leads to two subsystems: "odd modes" system and "even modes" system. The first subsystem has three resonance frequencies, $34.8 \mathrm{~Hz}, 137.6 \mathrm{~Hz}, 218.9 \mathrm{~Hz}$, and also three anti-resonance frequencies, $41.9 \mathrm{~Hz}, 140.5 \mathrm{~Hz}, 222.6 \mathrm{~Hz}$. On the other hand, the second subsystem has two main resonance frequencies, $69.2 \mathrm{~Hz}, 163.1 \mathrm{~Hz}$, and two anti-resonance frequencies, $89.2 \mathrm{~Hz}, 181.6 \mathrm{~Hz}$. Based on these experimental results the mathematical models of both subsystems can be determined by using the ARX method [23]. As a result, the estimate model of the subsystem may be expressed as

$$
\mathbf{G}(z)=\mathbf{M}^{-1}(z) \mathbf{L}(z)
$$

where $\mathbf{M}(z)=1+M_{1} z^{-1}+M_{2} z^{-2}+M_{3} z^{-3}+\ldots+M_{p} z^{-p}, \mathbf{L}(z)=L_{0}+L_{1} z^{-1}+L_{2} z^{-2}+L_{3} z^{-3}+\ldots+L_{p} z^{-p}$ and $p$ is the assumed order of the identified model.

The first step of the model identification is the determination of the model transfer function of the "odd modes" subsystem. For this purpose the order of the model is assumed in such a way that the difference between the model and the experimental data is not greater than $83 \%$, as is shown in fig. 13(a). However, the obtained order of the estimated model $p=55$ is definitely too high for the synthesis of the control system. So, in the next step, the discrete time model is changed into the continuous time model, and next transformed to the state space model. With the balance method the order of the model has been reduced to 20 retaining a very good fitting to the experimental data (fig. 13(b)). However the obtained model is still too high. Therefore, in the next step the zeros and the poles of equal frequencies are cancelled. As a result, the 6th order model is obtained as a minimum realization of the "odd modes" subsystem:

$$
G_{\text {odd }}(s)=\frac{Y_{\text {odd }}(s)}{U(s)}=0.0168 \cdot \frac{\left(s^{2}+11.23 s+6.482 e 4\right)\left(s^{2}+46.47 s+9.353 e 5\right)\left(s^{2}+62.66 s+2.685 e 6\right)}{\left(s^{2}+7.641 s+4.885 e 4\right)\left(s^{2}+36.7 s+8.443 e 5\right)\left(s^{2}+50.08 s+2.574 e 6\right)} .
$$


Next, the mathematical model of the "even modes" subsystem (the sensor located parallel to the $Y$-axis) is determined in a similar way. In this case, the assumed order model ensuring the $83 \%$ agreement between the estimated model and the experimental data is $p=65$ (fig. 14(a)). Using the balance method the order of the estimated model has been further reduced to 40 (fig. 14(b)). Finally, taking into account only the two lowest even resonance and anti-resonance frequencies the 4 th order model has been obtained. The final transfer function of the "even modes" subsystem is as follows:

$$
G_{\text {even }}(s)=\frac{Y_{\text {even }}(s)}{U(s)}=-0.00142 \cdot \frac{\left(s^{2}+6.32 s+3.273 e 5\right)\left(s^{2}+25.98 s+1.403 e 6\right)}{\left(s^{2}+22.84 s+1.896 e 5\right)\left(s^{2}+60.88 s+9.953 e 5\right)} .
$$

\section{Summary and conclusions}

The problem of the optimal location of the piezo-actuators and piezo-sensors for active vibration control of the rectangular aluminum plate with the SFSF boundary conditions is presented in the paper. Based on the Mindlin plate theory and the finite element analysis of the first five mode shapes and natural frequencies is conducted. Using the obtained results the equation of motion of the plate has been modified by introducing two bending moments $M_{x}(x, y)$ and $M_{y}(x, y)$ generated by the piezos in the two perpendicular directions $X$ and $Y$. The control forces generated by the piezos have been calculated for two different orientations of the piezos: parallel to the $X$-axis and parallel to the $Y$-axis. The results show that the values of the modal control forces significantly depend on the deflection of the plate, especially in the $X$-direction (figs. 5 and 6). Next, the performance index of modal control forces for two considered directions of vibration has been determined. Again, the obtained results have proven that the best locations of both piezo-actuators are at the vicinity of maximum values of the cost functions $J_{f \text {-odd }}$ and $J_{f \text {-even }}($ fig. 7$)$. Further analysis of the measurement signals has shown that also unit elongations $\varepsilon_{X}$ and $\varepsilon_{Y}$ depend on modal deflections of the plate. Moreover, these elongations depend on the orientations of the piezo-sensors on the plate. The elongations (fig. 9) have been used to determine performance indexes $J_{\varepsilon \text {-odd }}$ and $J_{\varepsilon \text {-even }}$ for odd and even modes, respectively. Again, maximum values of these cost functions have been used to determine the best locations of the piezo-sensors on the plate.

After numerical calculations the identification procedure of the plate equipped with two piezo-actuators and two piezo-sensors are carried out at the laboratory stand. In this way, the obtained results of the FEM analysis have been confirmed by the experimental investigations. The measurements have been conducted for two different orientations of the piezo-sensors on the plate. After several transformations of the experimental signals and by using the balance method the mathematical models $G_{\text {odd }}(\mathrm{s})$ and $G_{\text {even }}(\mathrm{s})$ have been obtained for two piezo-sensor locations: parallel to the $X$-axis and parallel to the $Y$-axis, respectively. In future research the separate control laws for odd and even modes of the considered plate will be designed especially for system with virtual collocation of the piezo-elements on the structure.

This work is financed from Statutory Work of Department of Automatic Control and Robotics S/WM/1/2016.

Open Access This is an open access article distributed under the terms of the Creative Commons Attribution License (http://creativecommons.org/licenses/by/4.0), which permits unrestricted use, distribution, and reproduction in any medium, provided the original work is properly cited.

\section{References}

1. B. Mladenovic, Z. Bonic, Facta Univ. 8, 2 (2010).

2. J. Lee, KSME Int. J. 17, 3 (2003).

3. A.K. Rao, S. Srinivas, Int. J. Solid Struct. 6, 1463 (1970).

4. R.H. Gutierrez, P.A. Laura, J. Sound Vibrat. 201, 133 (1997).

5. C.W. Lim, K.M. Liew, ASCE J. Eng. Mech. 121, 203 (1995).

6. H.B. Khurasia, S. Rawtani, J. Appl. Mech. 45, 215 (1978).

7. K.M. Liew, C.M. Wang, Y. Xiang, Vibration of Mindlin plates: programming the p-version Ritz method, 1st edition (Elsevier, Oxford, 1998).

8. J. Konieczny et al., Proceedings of Active 99: The International Symposium on Active Control of Sound and Vibration, Vol. 1, 2 (Florida, 1999).

9. A. Mystkowski, Mech. Syst. Signal Proces. 40, 783 (2013).

10. W. Raczka, M. Sibielak, J. Kowal, J. Low Frequency Noise Vibrat. Active Control 32, 1 (2013).

11. T. Baily, J.E. Hubbard, J. Guidance Control Dyn. 8, 605 (1985).

12. N.W. Hagood, E.F. Crawley, J. Guidance Control Dyn. 14, 490 (1991).

13. A.M. Sadri, J.R. Wright, R.J. Wynne, Smart Mater. Struct. 8, 521 (1991). 
14. T.L. Rocha et al., Proceedings of 11th International Congress on Sound and Vibration (Russia, 2004).

15. Z. Qiu, X. Zhang, H. Wu, H. Zhang, J. Sound Vibrat. 301, 521 (2007).

16. A. Koszewnik, Z. Gosiewski, Fast Prototyping of Active Vibration Damping System for Mechanical System, Mechanical System and Signal Processing, in Special Issue of Piezoelectric Technology, Vol. 36 (2013).

17. Z. Kulesza, J. Vibrat. Control 21, 8 (2015).

18. Z. Gosiewski, Z. Kulesza, Proceedings of 15th International Carpathian Control Conference, edited by I. Pietras, I. Podlubny, A. Nawrocka, B. Sapiński (Rytro, Poland, 2013).

19. A.J.M. Ferreira, Matlab Code for Finite Element Analysis: Solids and Structures (Springer, 2009).

20. Z. Osiński, Theory of Vibration (PWN, Warsaw, 1980) (in Polish).

21. A. Premount, Vibration Control of Active Structures, An Introduction, 2nd edition (Kluwer Academic Publisher, 2002).

22. M. Szmidt, Vibration analysis of rectangular plate with glued piezo-elements, PhD Thesis (Poland, Institute of Aviation Publisher, 2008) (in Polish).

23. J.N. Juang, Applied System Identification (Prentice-Hall, Inc., 2004). 